\title{
Variability and coding efficiency of noisy neural spike encoders
}

\author{
Peter N. Steinmetz*, Amit Manwani and Christof Koch \\ Computation and Neural Systems Program, 139-74, \\ California Institute of Technology, Pasadena, CA 91125. \\ $\{$ peter,quixote,koch\}@klab.caltech.edu
}

Keywords: Integrate-and-fire models, Hodgkin-Huxley model, stochastic ion channels, neuronal variability, signal estimation

*Author to whom correspondence should be addressed.

Submitted to Biosystems. Draft Copy, do not Distribute without PERMISSION. 


\begin{abstract}
Encoding synaptic inputs as a train of action potentials is a fundamental function of nerve cells. Although spike trains recorded in vivo have been shown to be highly variable, it is unclear whether variability in spike timing represents faithful encoding of temporally varying synaptic inputs or noise inherent in the spike encoding mechanism. It has been reported that spike timing variability is more pronounced for constant, unvarying inputs than for inputs with rich temporal structure. This could have significant implications for the nature of neural coding, particularly if precise timing of spikes and temporal synchrony between neurons is used to represent information in the nervous system.
\end{abstract}

To study the potential functional role of spike timing variability, we estimate the fraction of spike timing variability which conveys information about the input for two types of noisy spike encoders—an integrate and fire model with randomly chosen thresholds and a model of a patch of neuronal membrane containing stochastic $\mathrm{Na}^{+}$and $\mathrm{K}^{+}$channels obeying Hodgkin-Huxley kinetics. The quality of signal encoding is assessed by reconstructing the input stimuli from the output spike trains using optimal linear meansquare estimation. A comparison of the estimation performance of noisy neuronal models of spike generation enables us to assess the impact of neuronal noise on the efficacy of neural coding. The results for both models suggest that spike timing variability reduces the ability of spike trains to encode rapid time-varying stimuli. Moreover, contrary to expectations based on earlier studies, we find that the noisy spike encoding models encode slowly-varying stimuli more effectively than rapidly varying ones. 


\section{Introduction}

One of the fundamental computational roles of neurons is to encode their synaptic inputs into spike trains which are then communicated to other parts of the nervous system. The biophysical components of neurons (including synapses and a variety of ion channels) are inherently unreliable and behave probabilistically. Thus, the process of spike encoding is noisy and may result in variable timing of individual action potentials in response to identical inputs (Lecar \& Nossal, 1971a; Lecar \& Nossal, 1971b; Schneidman et al., 1998).

In vitro recordings in cortical pyramidal neurons have shown that spike timing reliability is higher for inputs which have rapid temporal modulations (Mainen \& Sejnowski, 1995). This argues in favor of the hypothesis that biophysical noise sources inherent in spike encoding may be a significant concern for slowly-varying input signals. The idea that neurons may encode signals with rapid temporal variation more accurately has also received theoretical support. Schneidman et al. simulated a $250 \mu \mathrm{m}^{2}$ patch of membrane containing stochastic Hodgkin and Huxley type $(\mathrm{HH}) \mathrm{Na}^{+}$and $\mathrm{K}^{+}$channels (Hodgkin \& Huxley, 1952) and showed that spike timing reliability was higher for rapidly changing input signals. (van Vreeswijk \& Sompolinsky, 1996; van Vreeswijk \& Sompolinsky, 1998). High spike timing reliability is intuitively appealing and can provide a substrate for temporal coding (Abeles, 1990; Bialek et al., 1991; Bialek \& Rieke, 1992; Richmond \& Optican, 1992; Softky \& Koch, 1993; Theunissen \& Miller, 1995). It is possible, however, that variability in spike timing represents faithful encoding of non-linear

or chaotic network dynamics rather than noise in the encoding neurons, especially in large networks of neurons

Our overall research goal is to assess the information-processing ability of neurons in terms of the respective efficacies of their biophysical components (synapse, dendritic tree, soma, and axon) by engaging in 
systematic analysis of the noise associated with each stage (Manwani \& Koch, 1998; Manwani \& Koch, 1999b; Manwani \& Koch, 1999a). We believe that the nature of information processing in biological systems, similar to their physical counterparts, is influenced by the magnitude and nature of their underlying noise sources. Thus, in order to understand how communication and computation processes work in the brain, it is important to study the sources of neuronal noise.

Here we quantify the effect of the variability of spike timing on the ability of neuronal spike encoding models to transmit information about their time-varying inputs. Within the context of a signal estimation paradigm, we use a statistical measure called the coding fraction to quantify the portion of variability in the output spike train which conveys information about the input, The goal in signal estimation is to estimate a random time-varying current injected into a spike encoding model from the corresponding spike train output and the coding fraction measures performance in the signal estimation task. In this paper, we examine the coding fraction for two noisy encoding models: an integrate-and-fire neuron with randomly chosen thresholds and a simulated patch of stochastic Hodgkin-Huxley channels. For both encoding models, we study the dependence of the coding fraction on the stimulus bandwidth and the mean firing rate of the neuron.

\section{Methods}

For the purpose of this paper, we will assume that the input to the spike encoder is given by $m(t)$, a time-varying continuous signal drawn from a Gaussian ensemble. The neuron's spiking mechanism transforms this input into a sequence of action potentials. This situation is shown schematically in figure 1. Though the models we consider here are highly simplified, the following analysis can be extended to more complicated encoding mechanisms which take into account non-linear aspects (like half-wave rectification or saturation) or temporal aspects (like plasticity or adaptation) of the neuron's 
input-output transformation.

The spike train output of the model neuron is denoted by $s(t)$ and is modeled as a sequence of delta functions corresponding the sequence of times $\left\{t_{i}\right\}$ when the model generates action potentials in response to the input $m(t)$. Thus, $s(t)$ can be written as,

$$
s(t)=\sum_{i} \delta\left(t-t_{i}\right)
$$

In the following, we consider two types of models which transform a continuous, time-varying signal into its corresponding spike train. Both these models are inherently noisy and thus, the spike trains resulting from repeated presentations of the same input are not identical.

\subsection{Integrate-and-fire model}

Integrate-and-fire models (I\&F) are phenomenological descriptions of spiking neurons (Stein, 1967a; Stein, 1967b; Tuckwell, 1988; Koch, 1999). Despite their simplicity, they retain two important aspects of neuronal firing, a subthreshold domain where the input to the neuron is passively integrated and a voltage threshold, which once exceeded, leads to the generation of stereotypical spikes.

The simplest version of the model, called the perfect integrate-and-fire model, comprises of a single capacitance $C$ followed by a fixed threshold $V_{\mathrm{th}}$. The model is a physiologically inaccurate abstraction of a real neuron but is often used for reasons of analytical tractability. If the input to the model is a current $m(t)$, the voltage output of the perfect integrator is given by,

$$
C \frac{d V(t)}{d t}=m(t)
$$

When the membrane voltage reaches $V_{\mathrm{th}}$, an impulse is generated and voltage is reset to zero. Mathematically, spike trains are modeled as a sequence of delta functions $\delta\left(t-t_{i}\right)$ where $t_{i}$ denotes the time 
instant at which the $i^{\text {th }}$ spike in the sequence occurs. For the perfect integrator, the successive times of spike occurrence $t_{i}$ can be obtained recursively from the equation,

$$
\int_{t_{i}}^{t_{i+1}} \mathrm{~d} t m(t)=C \cdot V_{\mathrm{th}}
$$

Neurons typically do not respond to their inputs instantaneously following an action potential. Due to biophysical considerations there is certain time interval following the generation of a spike during which another spike cannot be generated, irrespective of the strength of the input. This is called a refractory period and is usually denoted by $t_{\text {ref. }}$ In the case of the I\&F model, the effect of a refractory period can be replicated by holding the membrane potential at rest for a fixed duration $t_{\text {ref }}$ immediately after a spike.

The $\mathrm{f}-\mathrm{I}$ curve of the model in response to a constant current $I$ is linear, $f=I /\left(C . V_{\mathrm{th}}\right)$, where $f$ denotes the firing rate of the model in units of $\mathrm{Hz}$. The effect of the refractory period is to introduce non-linear saturation in the $\mathrm{f}-\mathrm{I}$ curve,

$$
f=\frac{I}{C \cdot V_{\mathrm{th}}+t_{\mathrm{ref}} I}
$$

The output of the perfect integrator to a constant current is a regular spike train with the time intervals between successive spikes exactly equal to $1 / f$. Real neurons seldom respond to a constant current input with a regular sequence of action potentials however. In fact, there is substantial variability in the exact timing of the spikes which is particularly pronounced in recordings in vivo (Holt et al., 1996).

A simple modification that allows the I\&F model to produce irregular spike trains in response to constant inputs is to consider the voltage threshold to be a random variable drawn from an arbitrary probability distribution $p\left(V_{\mathrm{th}}\right)$ (Holden, 1976; Gestri et al., 1980). We shall refer to this class as "integrate-and-fire models with random threshold".

By appropriate modification of parameters, this model can be used to mimic the random nature of 
neural spike trains. We assume $p\left(V_{\mathrm{th}}\right)$ is given by a gamma distribution of order $n$,

$$
p_{n}\left(V_{\mathrm{th}}\right)=c_{n}\left(\frac{V_{\mathrm{th}}}{\bar{V}_{\mathrm{th}}}\right)^{n-1} \exp \left(\frac{-n V_{\mathrm{th}}}{\bar{V}_{\mathrm{th}}}\right)
$$

with

$$
c_{n}=\frac{n^{n}}{(n-1) !} \frac{1}{\bar{V}_{\mathrm{th}}}
$$

where $\bar{V}_{\text {th }}$ denotes the mean voltage threshold. The order $n$ of the distribution determines the variability of the spike trains in response to a constant current injection.

A schematic diagram of the integrate and fire model with gamma distributed random threshold is shown in Figure 2A. For a perfect integrator, the interspike interval (denoted by $T$ ) is proportional to the voltage threshold. Thus, the resulting interspike intervals (ISIs) for the random threshold model are also gamma distributed around their mean value $\mu_{\mathrm{T}}$,

$$
\mu_{\mathrm{T}}=\frac{C \cdot \bar{V}_{\mathrm{th}}+t_{\mathrm{ref}} I}{I}
$$

The coefficient of variation of the ISI distribution, defined as the standard deviation of the length of the ISIs (denoted by $\sigma_{\mathrm{T}}$ ) divided by the mean ISI, is given by,

$$
\frac{\sigma_{\mathrm{T}}}{\mu_{\mathrm{T}}}=\frac{1}{\sqrt{n}}
$$

Thus, one can obtain spike trains of varying regularity by varying $n$. The case $n=1$ corresponds to a Poisson spike train which represents the most variable model in this class with a coefficient of variation equal to one. At the other extreme, $n \rightarrow \infty$ corresponds to the perfect integrate-and-fire model discussed above which has a coefficient of variation equal to zero.

Realistically speaking, the random voltage threshold model is not physiologically plausible since it is believed that the spiking mechanism in real neurons is very reliable (Calvin \& Stevens, 1968; Mainen \& Sejnowski, 1995). In the case of a perfect integrate-and-fire model, however, a random threshold can be 
shown to be equivalent to a random input current (Gestri et al., 1980). This random current may arise as a result of thermal noise, random ion-channel transitions or background synaptic activity (DeFelice, 1981; Manwani \& Koch, 1999a). In most cases, the random threshold model is mathematically and computationally easier to deal with than injecting a random input current (Gabbiani \& Koch, 1998). Thus, we will use the integrate and fire model with random threshold to phenomenologically model the variability commonly observed in spike trains of cortical neurons (Softky \& Koch, 1993; Holt et al., 1996; Shadlen \& Newsome, 1998).

\subsection{Stochastic Hodgkin-Huxley model}

Despite their analytical simplicity and widespread use, it must be noted that I\&F models are phenomenological models and cannot be used to faithfully describe biological neurons. Neuronal membranes contain several voltage- and ligand-gated ionic currents which are responsible for physiological properties which I\&F-like models fail to capture, such as the relative refractory period.

Hodgkin and Huxley (Hodgkin \& Huxley, 1952) successfully elucidated the ionic basis of neuronal excitability in the squid giant axon. Their mathematical model described the initiation and propagation of action potentials and enabled the analysis of a variety of neuronal membrane properties in terms of a description of ionic currents flowing across the membrane. In the original Hodgkin and Huxley model $(\mathrm{HH})$, macroscopic currents were expressed in terms of conductances due to the selective permeabilities of the membrane to different ionic species. These conductances, and the resulting membrane currents, are represented by deterministic and continuous variables. It is now known, however, that the deterministic macroscopic currents arise as a result of the summation of stochastic microscopic currents flowing through ion channels in the membrane.

Ion channels are protein macromolecules which switch randomly between discrete conformational states 
due to thermal agitation (Hille, 1992). HH-like voltage-gated ion-channels can be modeled as finite Markov chains where the conditional transition probabilities between the different conformational states are functions of the membrane voltage (Clay \& DeFelice, 1983; Strassberg \& DeFelice, 1993). The stochastic Markov version of the $\mathrm{HH}$ model converges to the classical, deterministic model as the number of channels grows large, but for realistic channel numbers, the stochastic model can exhibit a wide variety of behaviors (spontaneous spiking, bursting, chaos, and so on) that cannot be observed in deterministic model (DeFelice \& Isaac, 1992; White et al., 1998). It has been shown that channel transitions in stochastic $\mathrm{HH}$ model can potentially explain the level of precision of spike timing observed in cortical neurons (Schneidman et al., 1998).

We have analyzed the subthreshold voltage fluctuations of the stochastic $\mathrm{HH}$ model in a previous study (Steinmetz et al., 1999). Here we assess the influence of the stochastic nature of the spiking mechanism on its ability to encode time-varying inputs with the stochastic $\mathrm{HH}$ model described in (Steinmetz et al., 1999). In these simulations, the same band-limited white noise current which was used for the the I\&F model was injected into a patch of membrane containing stochastic $\mathrm{HH} \mathrm{Na}{ }^{+}$and $\mathrm{K}^{+}$channels. At each time step of the simulation, these channels change state probabilistically. The current for a given ionic species is determined by the corresponding number of channels in the open state. The total current entering the patch is integrated at every time step to determine the membrane voltage for the next time step and this process is repeated to obtain the voltage trajectory in response to a given time-varying input. For a detailed description of the stochastic model and Monte Carlo simulations see (Schneidman et al., 1998; Steinmetz et al., 1999). The membrane voltage is transformed into a sequence of spikes by counting as a spike occurrence each time the membrane voltage crosses a threshold level of $+10 \mathrm{mV}$ with a positive slope. A schematic diagram of the $\mathrm{HH}$ spike encoding model is shown in Figure $2 \mathrm{~B}$. 


\subsection{Optimal linear signal estimation}

The objective in optimal signal estimation is to recover the input $m(t)$ from the spike train $s(t)$, where the criterion for optimality is least mean-square-error (MSE). The optimal MSE estimator is intractable to derive and characterize analytically and so we shall restrict ourselves to optimal linear estimation.

As before, let $m(t)$ be a zero-mean random stimulus and $s(t)$ be the resulting spike train of a model neuron. $s(t)$ is assumed to be a point-process determined only by the sequence of spike times. We assume that $m(t)$ and $s(t)$ are jointly weak-sense stationary (WSS) processes with finite variances, $\left\langle m^{2}(t)\right\rangle=\sigma_{\mathrm{m}}^{2}\left\langle\infty,\left\langle|s(t)-\bar{\lambda}|^{2}\right\rangle\langle\infty\right.$, where $\bar{\lambda}=\langle s(t)\rangle$ is the mean firing rate of the neuron and $\langle\cdot\rangle$ denotes an average over the joint stimulus and spike train ensemble. Further, let

$$
\hat{m}(t)=g(t) \star s(t),
$$

denote the linear estimate of the input in terms of the spike train. $g(t)$ is the optimal linear filter which minimizes the MSE between the $m(t)$ and its estimate $\hat{m}(t)$ and $\star$ denotes the convolution operation. The MSE between the stimulus and the estimate is given by

$$
\mathcal{E}=\left\langle[m(t)-\hat{m}(t)]^{2}\right\rangle=\sigma_{\mathrm{m}}^{2}+\left\langle\hat{m}^{2}(t)\right\rangle-2\langle m(t) \hat{m}(t) \cdot\rangle
$$

The mathematical formulation and solution of the optimal linear estimation problem was originally carried out by Wiener (Wiener, 1949). Bialek and his colleagues introduced the "reconstruction approach" to theoretical neuroscience as a technique to quantify the amount of information single neurons can transmit about random inputs in the form of their spike outputs (Bialek et al., 1991; Bialek \& Rieke, 1992; Rieke et al., 1997). The reconstruction technique was successfully applied to quantify the efficacy of information-processing in peripheral neurons (Rieke et al., 1993; van Steveninck \& Bialek, 1995; Gabbiani et al., 1996; Rieke et al., 1997). For an extensive tutorial on the topic, see Gabbiani \& Koch 
(1998). The Fourier transform of the optimal linear estimation filter (denoted by $h_{\mathrm{opt}}(t)$ ) is given by

$$
H_{\mathrm{opt}}(f)=\frac{S_{\mathrm{ms}}(f)}{S_{\mathrm{ss}}(f)}
$$

where $S_{\mathrm{ms}}(f)$ denotes the cross-spectrum between the input and the spike train and $S_{\mathrm{ss}}(f)$ denotes the power spectrum of the spike train. The minimum MSE is given by

$$
\mathcal{E}_{\min }=\sigma_{\mathrm{m}}^{2}-\int_{-\infty}^{\infty} \mathrm{d} f \frac{\left|S_{\mathrm{ms}}(f)\right|^{2}}{S_{\mathrm{ss}}(f)}
$$

As in (Gabbiani \& Koch, 1998) we define a normalized, dimensionless measure called the coding fraction (denoted by $\xi$ ) which quantifies the fraction of the variability in the input that can be decoded from the spike train.

$$
\xi=1-\frac{\mathcal{E}_{\min }}{\sigma_{m}^{2}}
$$

The coding fraction varies between 0 and $1, \xi=1$ implies perfect reconstruction, whereas, $\xi=0$ implies performance at chance. Thus, the coding fraction can be used to assess the ability of spike generation mechanisms to encode time-varying inputs in the form of spike trains in the specific context of the signal estimation. A schematic diagram of the signal estimation paradigm is shown in Figure 1.

\section{Results}

We performed simulations for two noisy models of neuronal spike encoding, the I\&F model and the stochastic Hodgkin-Huxley model. For both models, we recorded the output spike times in response to the injection of pseudo-random, Gaussian, band-limited (flat power spectrum $S_{\mathrm{mm}}(f)$ over bandwidth $\left.B_{\mathrm{m}}\right)$ noise and computed the coefficient of variation (CV) of the interspike interval distribution and the coding fraction in the signal estimation task. CV is indicative of the variability of the spike train in response to the input, whereas the coding fraction quantifies the fraction of the variability in the spike train which enables the input to be accurately estimated. 
Figure 3 shows the $\mathrm{CV}$ for the two models as a function of the stimulus bandwidth $B_{\mathrm{m}}$ for a fixed mean firing rate. For the I\&F model, the mean firing rate $\bar{\lambda}$ depends only on the mean current $I$ injected into the neuron and can be determined by the $\mathrm{f}-\mathrm{I}$ curve (Eqn. 3). For the patch of stochastic $\mathrm{HH}$ channels, $\bar{\lambda}$ depends on several parameters like the area of the patch, standard deviation $\sigma_{\mathrm{m}}$ of the input and its bandwidth $B_{\mathrm{m}}$, in addition to the magnitude of the mean current $I$. For both models, $I$ was adjusted so that the mean firing rate $\bar{\lambda}$ was approximately equal to $50 \mathrm{~Hz}$. It can be seen that the $\mathrm{CV}$ decreases with increasing bandwidth for both spike encoding schemes. This result is in qualitative agreement with earlier experimental (Mainen \& Sejnowski, 1995) and computational (Schneidman et al., 1998) findings which demonstrated an inverse relationship between spike timing precision (using a measure different from $\mathrm{CV}$ ) and the temporal bandwidth of the input. Within the class of I\&F models, as expected, the $\mathrm{CV}$ is lower for models with higher $n$ which fire more regularly.

The dependence of the coding fraction on the stimulus bandwidth for the two models (for a fixed mean firing rate $\bar{\lambda}=50 \mathrm{~Hz}$ ) is shown in Figure 4 . For both encoding mechanisms, the coding fraction decreases as the stimulus bandwidth is increased. This is contrary to expectation; since the variability of spike timing increases with stimulus bandwidth, one would expect that this would lead to better performance in the encoding task. However, we find that these noisy spike encoding models encode slowly-varying stimuli more effectively than rapid ones. The similar dependence of $\mathrm{CV}$ and coding fraction on stimulus bandwidth suggests that the variability represents faithful encoding of the input modulations. On the other hand, the variability due to the randomness in the threshold is a form of noise, and as expected, the coding fraction is higher for I\&F models with larger $n$. An additional point, shown in this figure overall, is that for all of the noisy encoders (that is, for $n<\infty$ ), the coding fraction is less than 0.5 ; that is, the majority of variability in spike timing reflects noise, rather than variations in the input.

Next we explored how $\mathrm{CV}$ and the coding fraction depend on the mean firing rate of the model neurons. 
For both models, we changed the mean current $I$ to vary the firing rate while maintaining constant contrast of the input. The contrast was defined as $c=\sigma_{\mathrm{m}} / I$ and set to $c=0.33$. Figures 5 and 6 show the $\mathrm{CV}$ and the coding fraction, respectively, as a function of mean firing rate $\bar{\lambda}$. For the I\&F model, both the coding fraction and CV increase monotonically with firing rate. Again, this implies that the variability in the output spike train represents faithful encoding of the input modulations, and thus, greater variability enables better signal estimation. For the $\mathrm{HH}$ model, the opposite trend holds for the $\mathrm{CV}$, which decreases with increasing mean firing rate. This trend probably reflects a loss of shorter inter-spike intervals at higher firing rates and is a consequence of the refractoriness of the $\mathrm{HH}$ model. This variance in the dependence of the $\mathrm{CV}$ on the firing rate possibly points to a fundamental difference between the two encoding models and we are currently investigating this issue further. On the other hand, the coding fraction for the $\mathrm{HH}$ model also decreases with increasing firing rate, similarly to the trend with the I\&F model.

To further explore the dependence of the coding fraction on the firing rate, we plot coding fraction of the I\&F model (different $n$ ) as a function of the $\bar{\lambda} / B_{\mathrm{m}}$ ratio for different combinations of the mean firing rate and input bandwidth in figure 7 . The results demonstrate that the coding fraction depends on this ratio. The ratio $\bar{\lambda} / B_{\mathrm{m}}$ represents the number of spike per stimulus time constant; the larger the number of spikes available in a time interval over which the input is relatively constant, the better an estimate of the neuron's instantaneous firing rate and thus, the better an estimate of the instantaneous value of the input. This can be intuitively expected due to the linear relationship between the input current and the firing rate for the I\&F model. Thus, estimating the input is equivalent to estimating the instantaneous firing rate of the model. This is not true for the $\mathrm{HH}$ model, however, and consequently the coding fraction depends on the absolute values of $\bar{\lambda}$ and $B_{\mathrm{m}}$ and not on the ratio $\bar{\lambda} / B_{\mathrm{m}}$ alone (data not shown). 


\section{Discussion}

Deciphering the neural code requires an understanding of the biophysical constraints which limit the temporal precision of neuronal spike trains. It remains unclear whether cortical neurons behave as integrators and respond to the summed activity of hundreds of synaptic inputs on the time scale of tens or hundreds of milliseconds (Shadlen \& Newsome, 1994; Shadlen \& Newsome, 1998) or as temporal coincidence detectors (Abeles, 1990; Softky, 1995) and are sensitive to the simultaneous arrival of a handful of strategic synaptic inputs on a millisecond time scale. In a functioning network of neurons, the synaptic inputs to a single neuron are rapidly fluctuating and the resulting currents appear noisy (Destexhe \& Pare, 1999). In this environment, variability in the timing of the spikes output by a single neuron may represent either this fluctuating input or noise sources inherent in the neuron and the process of synaptic transmission. In this paper we used the coding fraction to quantify the fraction of variability conveying information about the input for two types of noisy spike encoders-an integrate and fire model with randomly chosen thresholds and a patch of simulated stochastic Hodgkin-Huxley $\mathrm{Na}^{+}$and $\mathrm{K}^{+}$channels.

For both types of noisy encoders, the coding fraction is higher for smaller stimulus bandwidths. This result is distinct from viewing the question in terms of spike timing reliability and precision. Both reliability and precision decrease for smaller stimulus bandwidths. Thus, the coding fraction results suggest that noisy spike encoders are likely to encode slowly varying stimuli more efficiently than rapidly varying stimuli. This is in contrast to earlier results (Mainen \& Sejnowski, 1995; Schneidman et al., 1998) which used spike timing reliability as a measure of the quality of neural encoding.

The differences between these approaches reflect different assumed goals for computation within the

nervous system. Measuring spike timing reliability and precision implicitly assumes that the times of 
individual spikes are meaningful and convey information. Measuring the coding fraction assumes that knowledge of the time course of the input signal is the critical variable. Our own view is that this issue cannot be definitely resolved until the relevant neural codes are determined and that, in the interim, the coding fraction represents a useful general approach.

These theoretical results can be tested experimentally by performing recordings similar to those of Mainen \& Sejnowski (1995). For this test, one would again inject current into a cell in vitro, but analyze the coding fraction of the output, rather than spike timing and precision (for strict correspondence with these simulations, a band-limited white noise stimulus should be used rather than white noise filtered by alpha functions, but this is unlikely to constitute a significant difference.) Preliminary results of the coding fraction in cortical pyramidal cells and interneurons have been reported by Fellous et al., for sinusoidally varying stimuli, but a detailed comparison will require further analysis due to differences in the stimulating paradigm.

When interpreting these results, two general limitations must be borne in mind. First, the coding fraction is computed using white noise as an input. The actual neural code used by cortical pyramidal neurons is presently unknown and is quite likely to vary between different areas in the brain. Thus, we have chosen to use band-limited white noise as an input to provide a general indication of performance in encoding signals which have power distributed over a range of frequencies. The inherent problem with this approach, however, is its broad coverage of possible input signals. If the nervous system is highly tuned to one specific input pattern or has been optimized to process natural and ecologically relevant signals with specific statistical properties, white noise may not represent these signals accurately. Thus, the coding fraction for white noise stimuli must be interpreted as an indicator of average performance over a broad range of possible input signals.

The second general limitation of examining the coding fraction is that it represents the performance of 
a linear estimator. It does not capture the performance characteristics of non-linear encoders in general. Bialek et al. (Rieke et al., 1997), have shown that in most cases the difference in performance between linear and non-linear estimators is marginal though. Thus, if the mean rate of neuronal firing, or some function of it is the representational variable, the coding fraction examined here is likely to be a good indicator of coding efficiency.

Another limitation of this study is that it examines coding efficiency for a single neural encoder and thus does not reflect information which may be conveyed by specific population codes, such as temporal synchrony between neurons. This type of question will require further study with populations of encoders acting on variably synchronized inputs.

As shown by the results for the integrate-and-fire models with varying noise levels and by patches with stochastic channels of varying area, the amount of noise in the encoder affects the exact values of both the CV and the coding fraction. We are currently investigating the effect of parameters like the membrane patch area, temperature as well as the nature of the kinetic scheme on the efficiency of encoding. For all of the noisy encoders examined here, however, the overall conclusion remains that these spike encoders are more efficient for slowly varying stimuli, in the sense that the coding fraction is higher. From this perspective, noisy spike encoders in realistic environments have a preference for inputs which vary slowly in time.

\section{Acknowledgements}

This work was funded by NSF, NIMH and the Sloan Center for Theoretical Neuroscience at Caltech. We would like to thank our collaborators Michael London, Elad Schneidman and Idan Segev for their invaluable suggestions. 


\section{References}

Abeles M., 1990. Corticonics: Neural Circuits of the Cerebral Cortex. Cambridge University Press: Cambridge, United Kingdom.

Bialek W., Rieke F., 1992. Reliability and information-transmission in spiking neurons. Trends Neurosci. 15, 428-434.

Bialek W., Rieke F., van Steveninck R.R.D., Warland D., 1991. Reading a neural code. Science 252, 1854-1857.

Calvin W.H., Stevens C.F., 1968. Synaptic noise and other sources of randomness in motoneuron interspike intervals. J. Neurophysiol. 31, 574-587.

Clay J.R., DeFelice L.J., 1983. Relationship between membrane excitability and single channel open- close kinetics. Biophys. J. 42, 151-157.

DeFelice L.J., 1981. Introduction to Membrane Noise. Plenum Press: New York, New York.

DeFelice L.J., Isaac A., 1992. Chaotic states in a random world. J. Stat. Phys. 70, 339-352.

Destexhe A., Pare D., 1999. Impact of network activity on the integrative properties of neocortical pyramidal neurons in vivo. J. Neurophysiol, 81, 1531-1547.

Gabbiani F., Koch C., 1998. Principles of spike train analysis. In: Koch C., Segev I. (eds.), Methods in Neuronal Modeling: From lons to Networks, second edn., MIT Press: Cambridge, Massachusetts.

Gabbiani F., Metzner W., Wessel R., Koch C., 1996. From stimulus encoding to feature extraction in weakly electric fish. Nature 384, 564-567.

Gestri G., Mastebroeck H.A.K., Zaagman W.H., 1980. Stochastic constancy, variability and adaptation of spike generation: Performance of a giant neuron in the visual system of the fly. Biol. Cybern. 38, 31-40.

Hille B., 1992. Ionic Channels of Excitable Membranes. Sinauer Associates: Sunderland, Massachusetts.

Hodgkin A.L., Huxley A.F., 1952. A quantitative description of membrane current and its application to conductation and excitation in nerve. J. Physiol. (Lond.) 117, 500-544. 
Holden A.V., 1976. Models of the Stochastic Activity of Neurones. Springer Verlag: New York, New York.

Holt G.R., Softky W.R., Koch C., Douglas R.J., 1996. Comparison of discharge variability in vitro and in vivo in cat visual cortex neurons. J. Neurophysiol. 75, 1806-1814.

J.-M. Fellous R. P. N. Rao A.R.H., Sejnowski T.J., 1999. Spike timing reliability in the prefrontal cortex depends on the frequency content of its synaptic inputs. In: Soc. Neurosci. Abstr..

Koch C., 1999. Biophysics of Computation: Information Processing in Single Neurons. Oxford University Press, New York, New York.

Lecar H., Nossal R., 1971a. Theory of threshold fluctuations in nerves. I. Relationships between electrical noise and fluctuations in axon firing. Biophys. J. 11, 1048-1067.

Lecar H., Nossal R., 1971b. Theory of threshold fluctuations in nerves. II. Analysis of various sources of membrane noise. Biophys. J. 11, 1068-1084.

Mainen Z.F., Sejnowski T.J., 1995. Reliability of spike timing in neocortical neurons. Science 268, 1503-1506.

Manwani A., Koch C., 1998. Synaptic transmission: An information-theoretic perspective. In: Jordan M., Kearns M.S., Solla S.A. (eds.), Advances in Neural Information Processing Systems 10, pp 201-207. MIT Press: Cambridge, Massachusetts.

Manwani A., Koch C., 1999a. Detecting and estimating signals in noisy cable structures: I. Neuronal noise sources. Neural Comput. 11, 1797-1829.

Manwani A., Koch C., 1999b. Signal detection in noisy weakly-active dendrites. In: Kearns M.S., Solla S.A., Cohn D.A. (eds.), Advances in Neural Information Processing Systems 11, MIT Press: Cambridge, Massachusetts.

Richmond B.J., Optican L.M., 1992. The structure and interpretation of neuronal codes in the visual system. In: Wechsler H. (ed.), Neural Networks for Perception, pp. 104-119, Academic Press.

Rieke F., Warland D., Bialek W., 1993. Coding efficiency and information rates in sensory neurons. Europhysics Lett. 22, 151-156.

Rieke F., Warland D., van Steveninck R.D.R., Bialek W., 1997. Spikes: Exploring the Neural Code. MIT Press: Cambridge, Massachusetts. 
Schneidman E., Freedman B., Segev I., 1998. Ion-channel stochasticity may be critical in determining the reliability and precision of spike timing. Neural Comput. 10, 1679-1703.

Shadlen M.N., Newsome W.T., 1994. Noise, neural codes and cortical organization. Curr. Opin. Neurobiol. 4, 569-579.

Shadlen M.N., Newsome W.T., 1998. The variable discharge of cortical neurons: implications for connectivity, computation, and information coding. J. Neurosci. 18, 3870-3896.

Softky W.R., 1995. Simple codes versus efficient codes. Curr. Opin. Neurobiol. 5, 239-247.

Softky W.R., Koch C., 1993. The highly irregular firing of cortical cells is inconsistent with temporal integration of random epsps. J. Neurosci. 13, 334-350.

Stein R.B., 1967a. The frequency of nerve action potentials generated by applied currents. Proc. R. Soc. Lond. B 167, 64-86.

Stein R.B., 1967b. Some models of neuronal variability. Biophys. J. 7, 37-68.

Steinmetz P.N., Manwani A., London M., Koch C., 1999. Sub-threshold voltage noise due to channel fluctuations in active neuronal membranes. J. Comput. Neurosci. In press.

Strassberg A.F., DeFelice L.J., 1993. Limitations of the Hodgkin-Huxley formalism: effect of single channel kinetics on transmembrane voltage dynamics. Neural Comput. 5, 843-855.

Theunissen F., Miller J.P., 1995. Temporal encoding in nervous systems: a rigorous definition. J. Comput. Neurosci. $2,149-162$.

Tuckwell H.C., 1988. Introduction to Theoretical Neurobiology II: Nonlinear and Stochastic Theories. Cambridge University Press: New York, New York.

van Steveninck R.D., Bialek W., 1995. Reliability and statistical efficiency of a blowfly movement-sensitive neuron. Phil. Trans. Roy. Soc. Lond. B 348, 321-340.

van Vreeswijk C., Sompolinsky H., 1996. Chaos in neuronal networks with balanced excitatory and inhibitory activity. Science $274,1724-6$. 
van Vreeswijk C., Sompolinsky H., 1998. Chaotic balanced state in a model of cortical circuits. Neural Comput. 10, 1321-1371.

White J.A., Klink R., Alonso A., Kay A.R., 1998. Noise from voltage-gated ion channels may influence neuronal dynamics in the entorhinal cortex. J. Neurophysiol. 80, 262-269.

Wiener N., 1949. Extrapolation, Interpolation and Smoothing of Stationary Time Series. MIT Press: Cambridge, Massachusetts. 


\section{Figures}

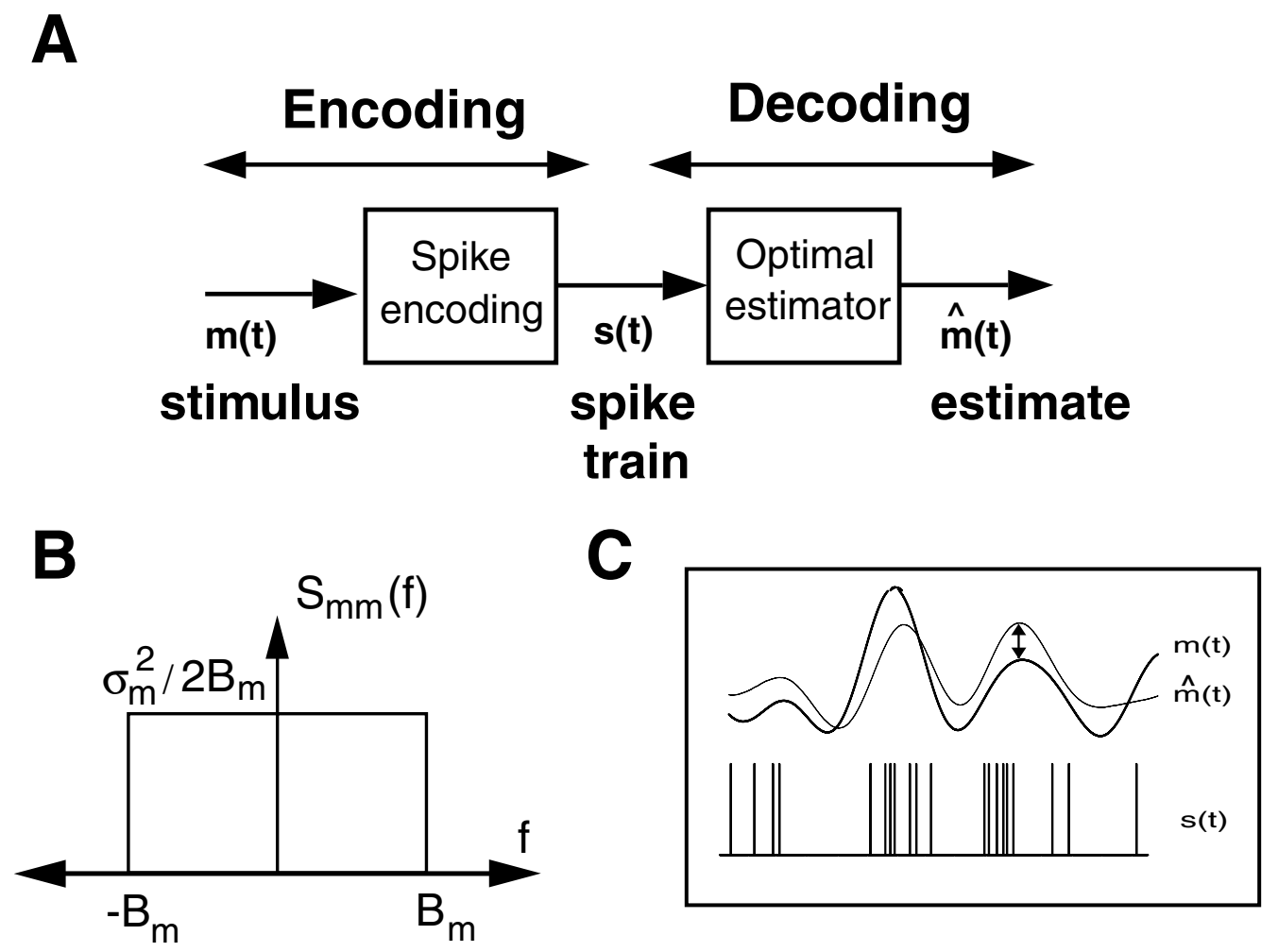

Figure 1: Block diagram of the signal estimation paradigm.

A: A time-varying input stimulus $m(t)$ is injected into noisy neural spike encoding model which is transformed into an output spike train $s(t)$. Linear systems theory is used to derive the optimal linear estimate, $\hat{m}(t)$ of the input from the spike train. B: The standard deviation of the Gaussian, band-limited, wide sense stationary (WSS) stochastic process $m(t)$ is denoted by $\sigma_{\mathrm{m}}$. Its power spectrum $S_{\mathrm{mm}}(f)$ is flat over a bandwidth $B_{\mathrm{m}}$. C: Representative examples of the time-varying input signal $m(t)$, the spike train output of the noisy encoder $s(t)$, and the optimal linear reconstruction of input $\hat{m}(t)$. 

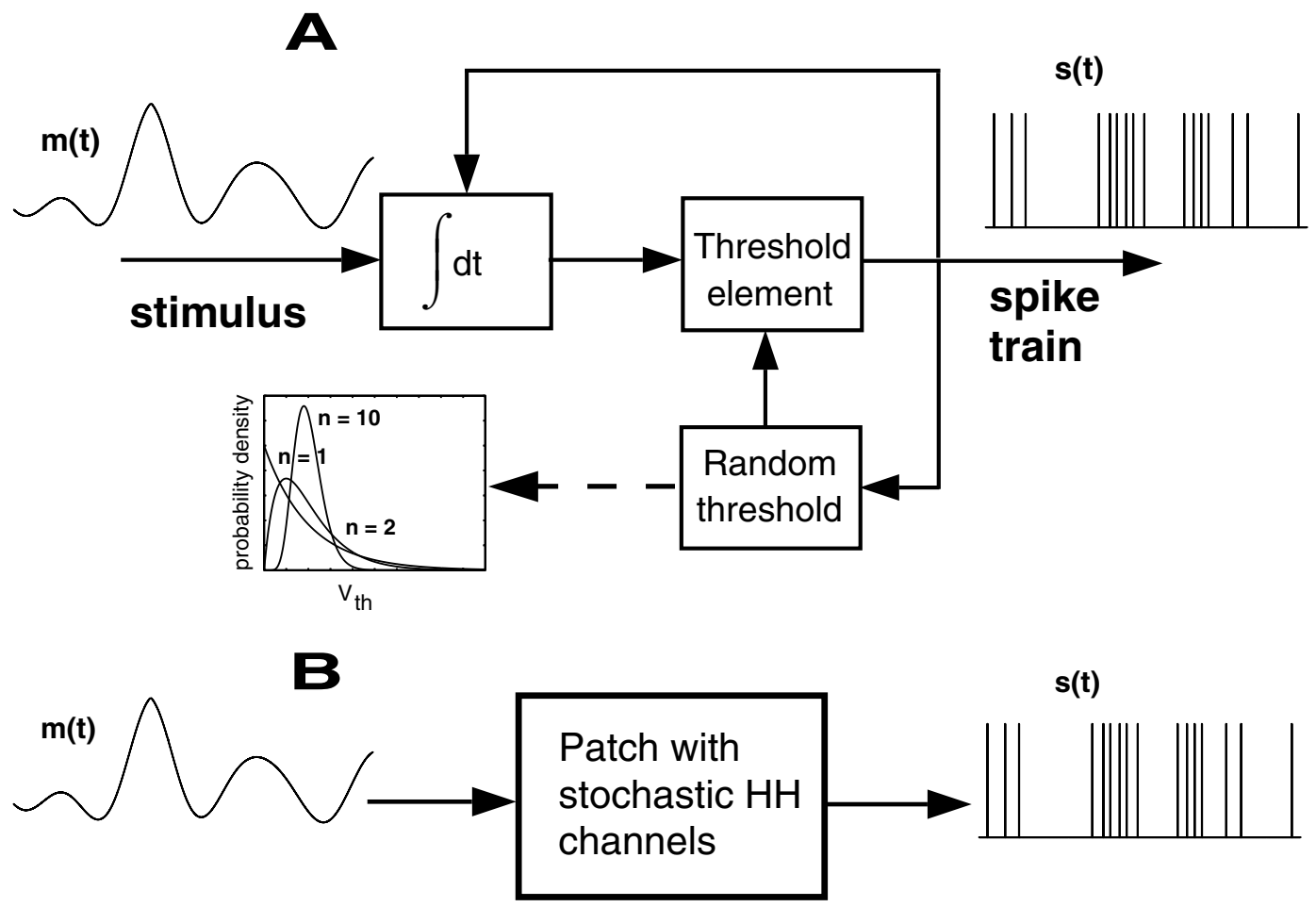

Figure 2: Schematic diagrams of noisy spike encoders.

A: Integrate and fire model with random threshold. The time-varying stimulus $m(t)$ is integrated over time and when the output exceeds a randomly chosen threshold, a spike is generated and the integrator is reset. Voltage thresholds are drawn from an $n$th order gamma distribution where $n$ determines the variability in spike timing (inset shows gamma distributions for $\mathrm{n}=1,2$ and 10). Model parameters: $\bar{V}_{\mathrm{th}}=20 \mathrm{mV}, \mathrm{C}=0.5 \mathrm{nF}, \Delta \mathrm{t}=$ $0.5 \mathrm{msec}, t_{\mathrm{ref}}=0.5 \mathrm{msec}$. B: Membrane patch with stochastic voltage-gated ion channels. The time-varying input current is injected into a patch of membrane containing stochastic Hodgkin-Huxley type $\mathrm{Na}^{+}$and $\mathrm{K}^{+}$channels. When the membrane voltage exceeds $10 \mathrm{mV}$ above rest, a spike is recorded in the output spike train $s(t)$. Model parameters: patch area $A=1000 \mu \mathrm{m}^{2}, \mathrm{Na}^{+}$channel conductance $=20 \mathrm{pS}, \mathrm{K}^{+}$channel conductance $=20 \mathrm{pS}$, $\mathrm{Na}^{+}$density $=60$ channels $/ \mu \mathrm{m}^{2}, \mathrm{~K}^{+}$density $=18$ channels $/ \mu \mathrm{m}^{2}, \Delta t=5 \mu \mathrm{sec}, T=6.3^{\circ} \mathrm{C}$. 


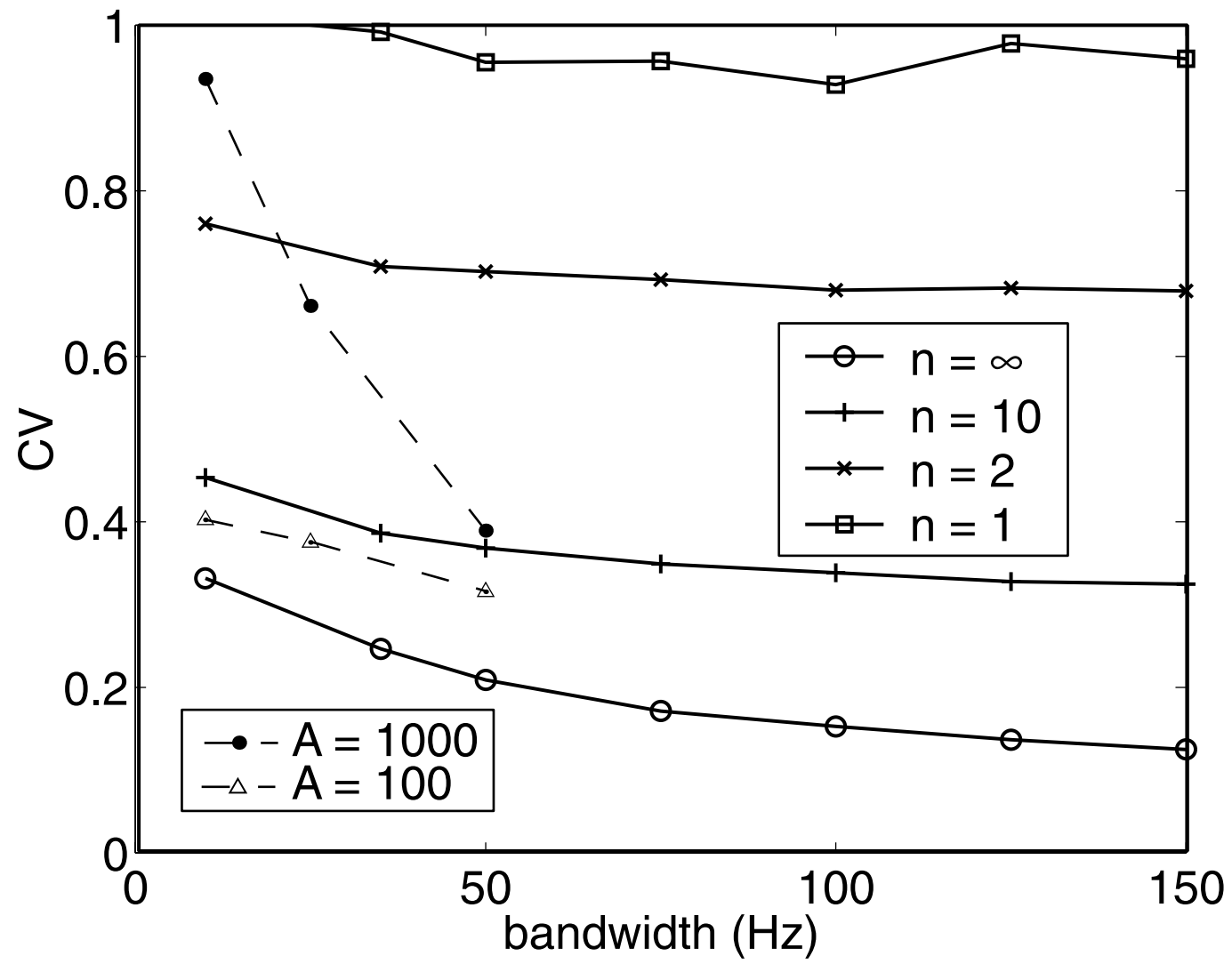

Figure 3: Spike variability vs. stimulus bandwidth.

Coefficient of variability (CV) of the interspike interval distribution (ISI) as a function of stimulus bandwidth for the I\&F and HH models. The solid lines show the CV for the I\&F model for different orders of the gamma distributed voltage threshold, whereas the dashed lines represent the $\mathrm{HH}$ model for two different patch areas. The mean firing rate $\bar{\lambda}$ for both the models was adjusted to be equal to $50 \mathrm{~Hz}$ by injecting a constant current in addition to the time-varying input. The contrast of the input, defined as the standard deviation $\sigma_{\mathrm{m}}$ divided by the mean $I$, was equal to 0.33 in both cases. 


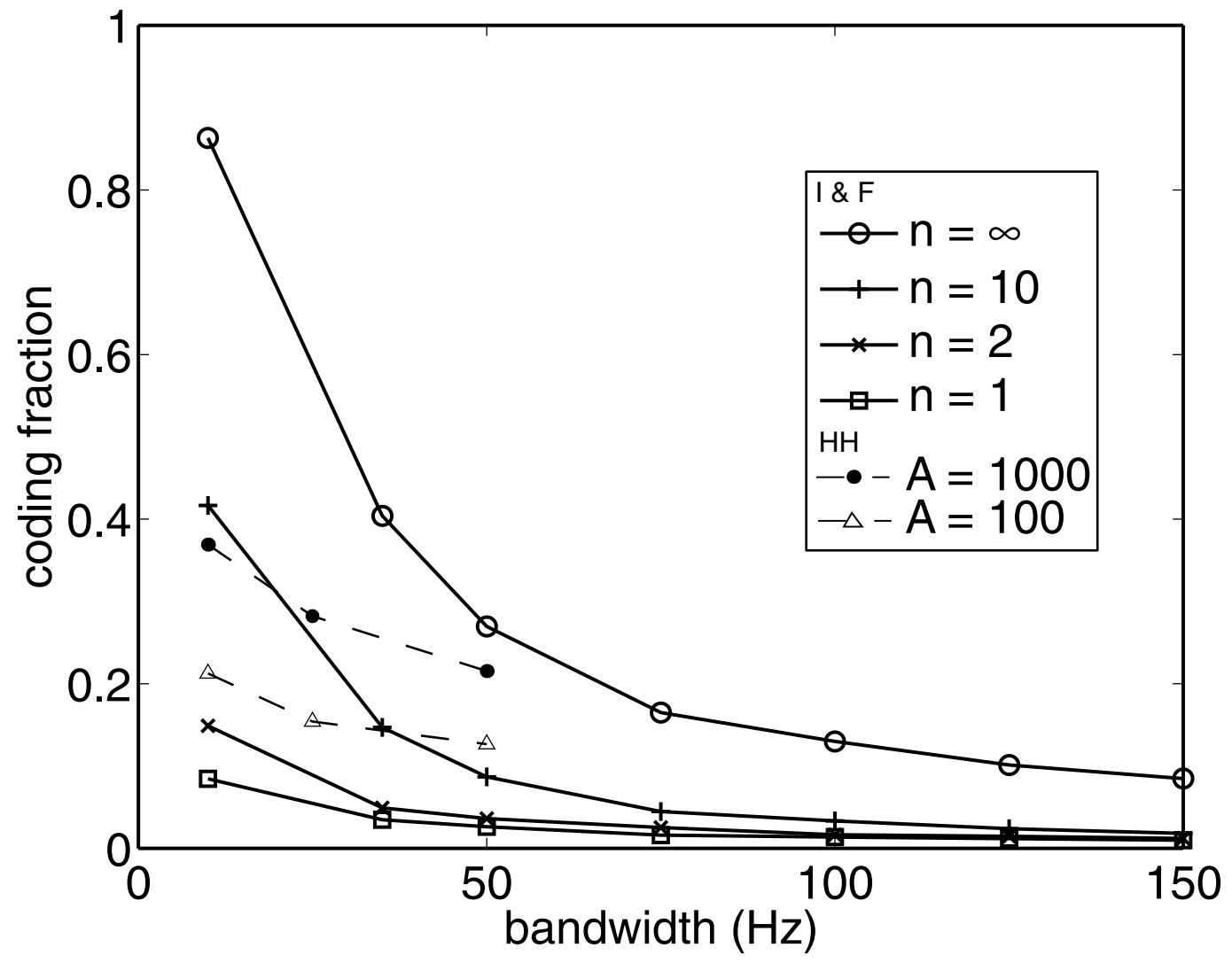

Figure 4: Coding efficiency vs. stimulus bandwidth.

Coding fraction as a function of stimulus bandwidth $B_{\mathrm{m}}$ for the I\&F and $\mathrm{HH}$ models. The solid lines show the coding fraction for the I\&F model for different orders, $n$, of the gamma distributed voltage threshold (ranging from a perfectly regular I\&F model to a Poisson model), whereas, the dashed lines indicate the corresponding coding fraction for the $\mathrm{HH}$ model for two different patch areas. The mean firing rate $\bar{\lambda}$ for both the models was adjusted to be equal to $50 \mathrm{~Hz}$ by injecting a constant current $I$ in addition to the time-varying input. As in figure 3 , the contrast of the input was equal to 0.33 in both cases. 


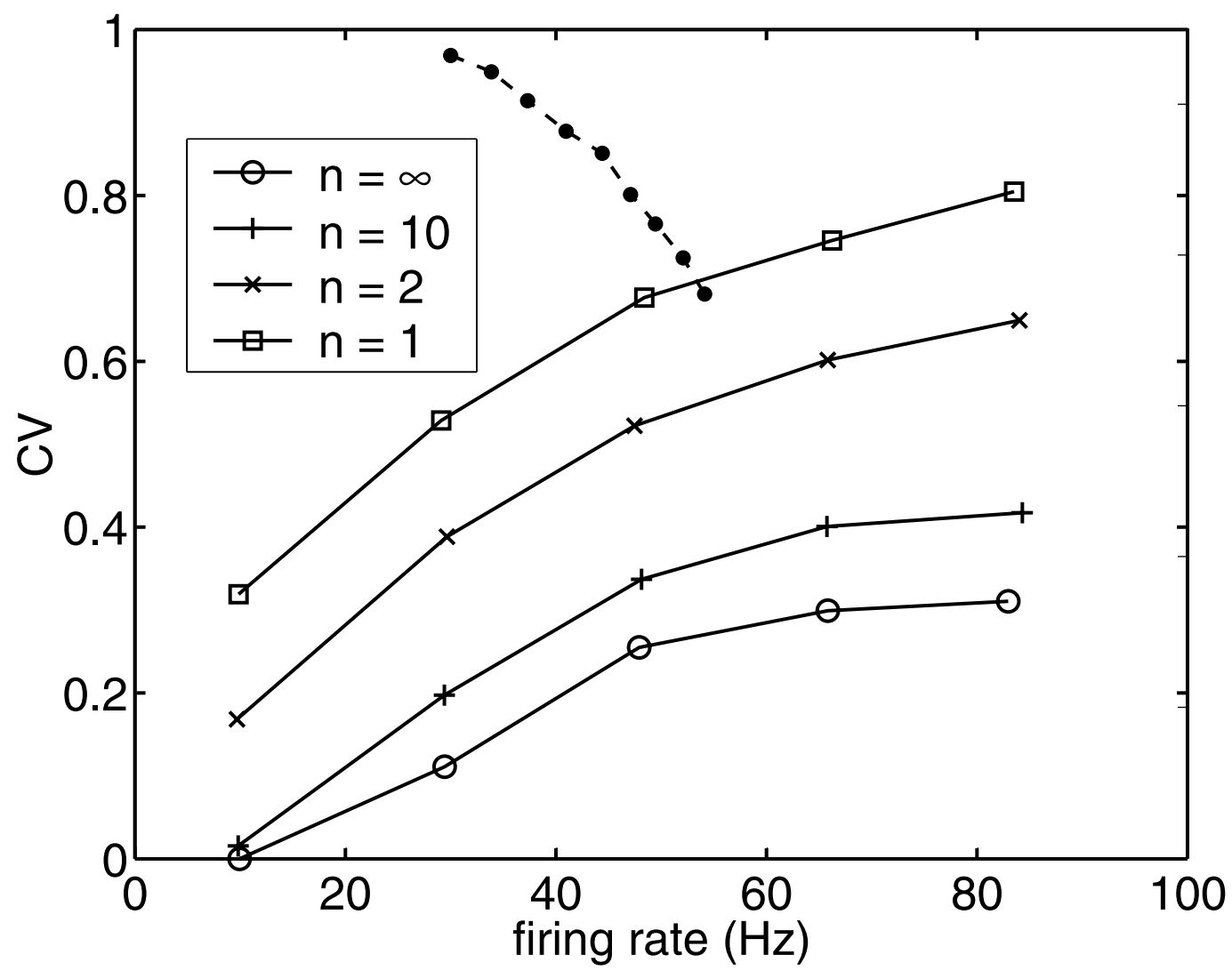

Figure 5: Spike variability vs. mean firing rate.

CV of the ISI distribution for the I\&F and $\mathrm{HH}$ models as a function of mean firing rate $\bar{\lambda}$. The solid lines correspond to the I\&F model for different orders $n$ of the gamma distributed threshold. The dashed line shows the corresponding coding fraction for a $1000 \mu \mathrm{m}^{2}$ patch with stochastic $\mathrm{HH}$ channels. Input parameters: bandwidth $B_{\mathrm{m}}=10 \mathrm{~Hz}$, contrast $=0.33$. 


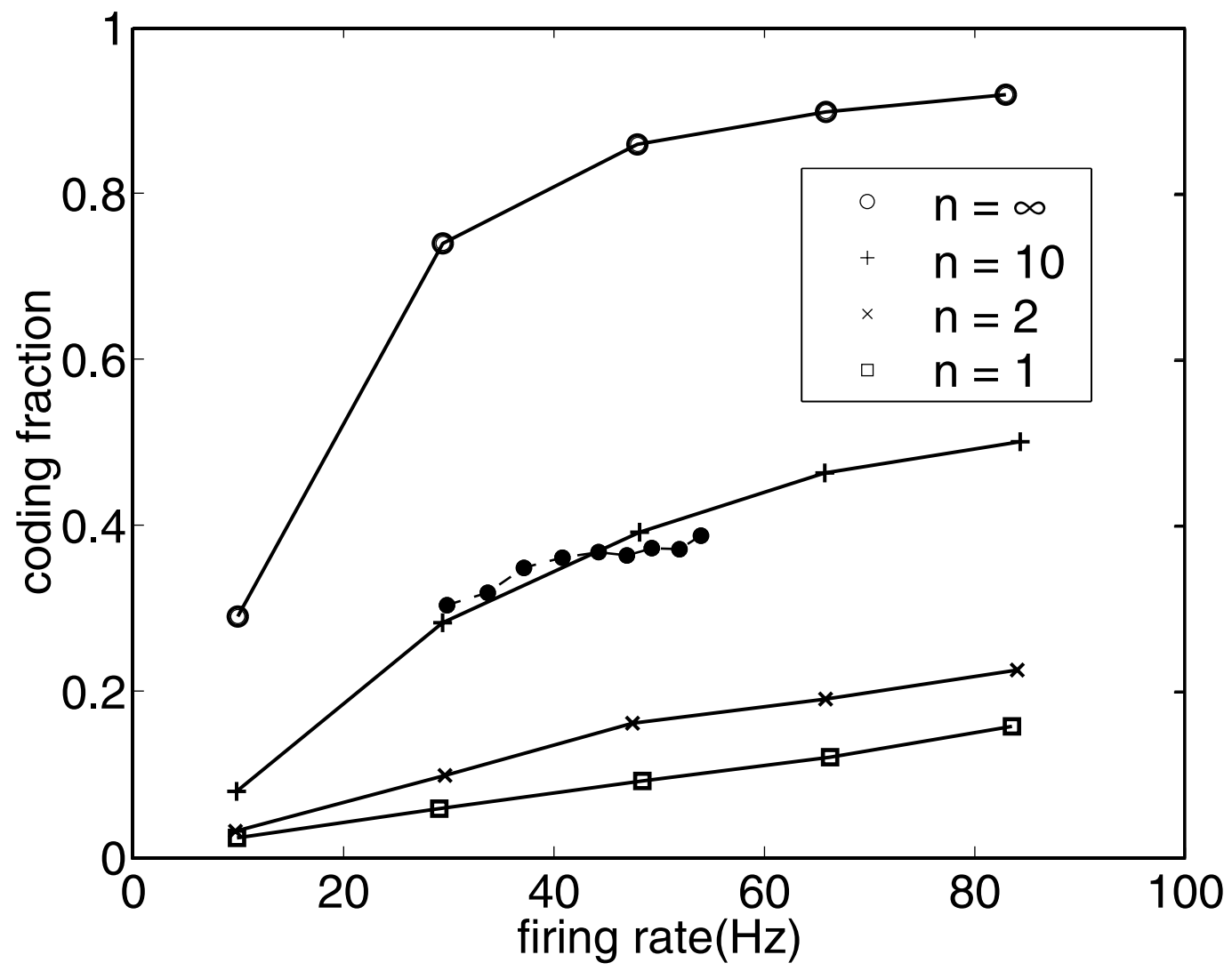

Figure 6: Coding efficiency vs. mean firing rate.

Coding fraction for the signal estimation task as a function of the mean firing rate $\bar{\lambda}$ for the I\&F and HH models.

The solid lines correspond to the I\&F model for different orders $n$ of the gamma distributed threshold, whereas the dashed line corresponds to a $1000 \mu \mathrm{m}^{2}$ patch containing stochastic $\mathrm{HH}$ channels. Input parameters: bandwidth $B_{\mathrm{m}}=10 \mathrm{~Hz}$, contrast $=0.33$. 


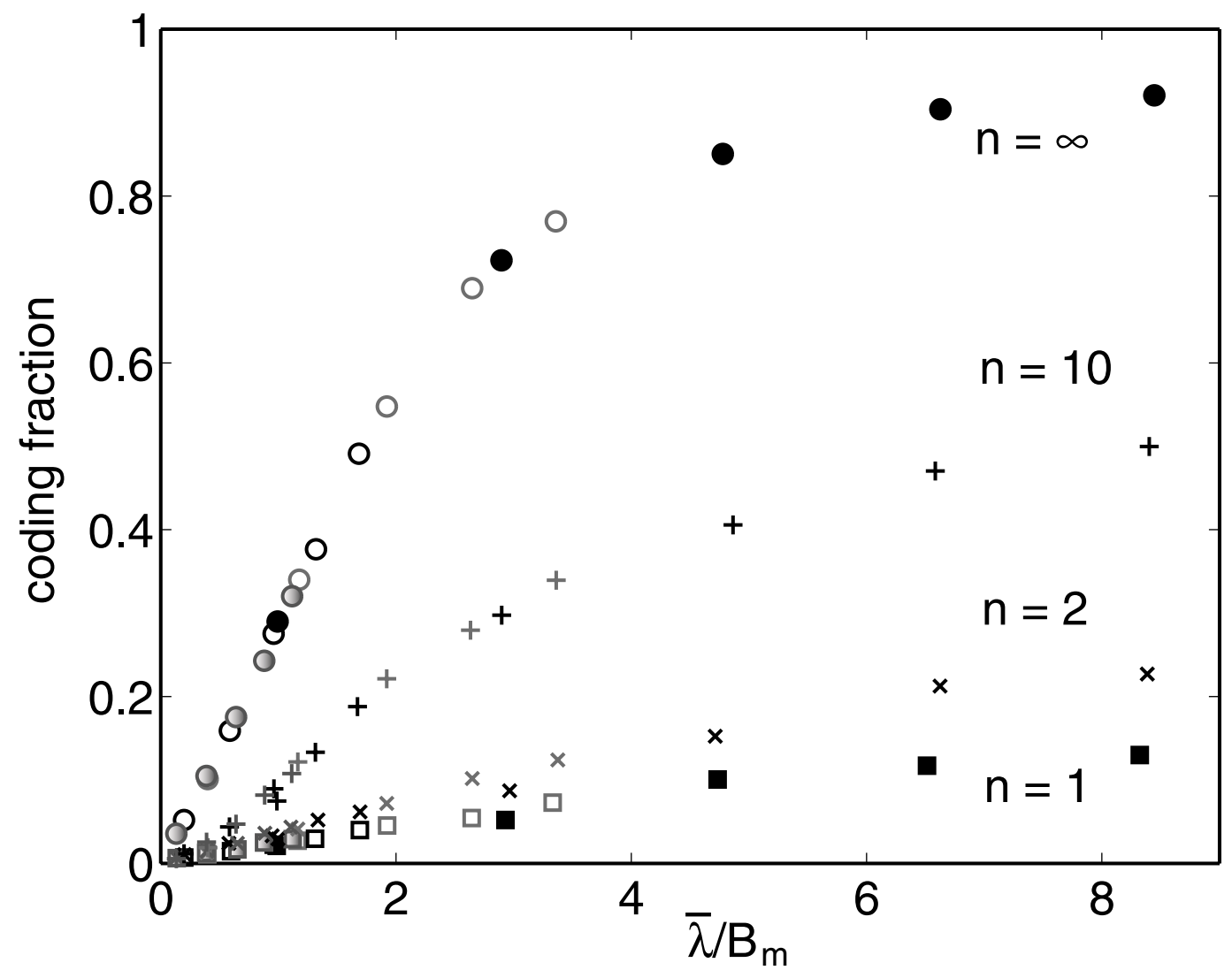

Figure 7: Coding fraction vs. ratio of mean firing rate and input bandwidth.

The coding fraction for the integrate and fire model with random threshold as a function of the ratio of the mean firing rate $\bar{\lambda}$ to the input bandwidth $B_{\mathrm{m}}$. The symbols represent results for different levels of noise in the encoder, denoted by the order of the voltage threshold gamma distribution. The different shades of gray correspond to different combinations of input bandwidth $B_{\mathrm{m}}$ and mean firing rate $\bar{\lambda}$. The contrast of the input is 0.33 . 